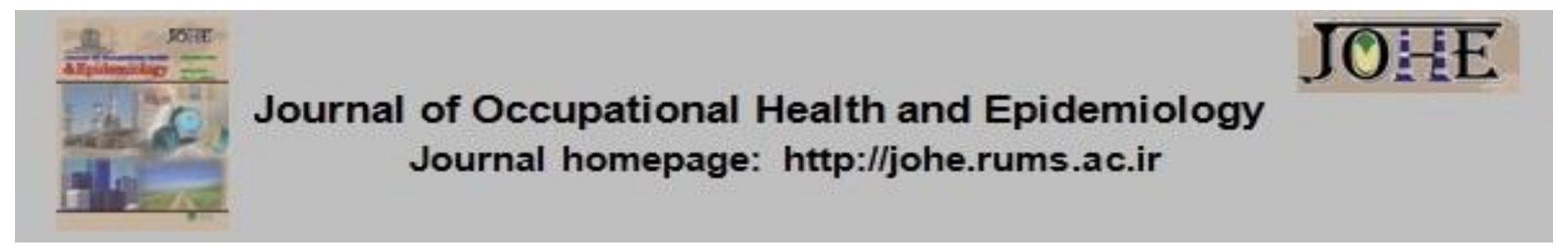

\title{
Association between prospective, retrospective memories and job performance of nurses in the city of Ahvaz, Iran, in 2016
}

\author{
Zahra Ebadi ${ }^{1 *}$ \\ - Assistant Prof., Department of Psychology, University of Payame Noor, Tehran, Iran.
}

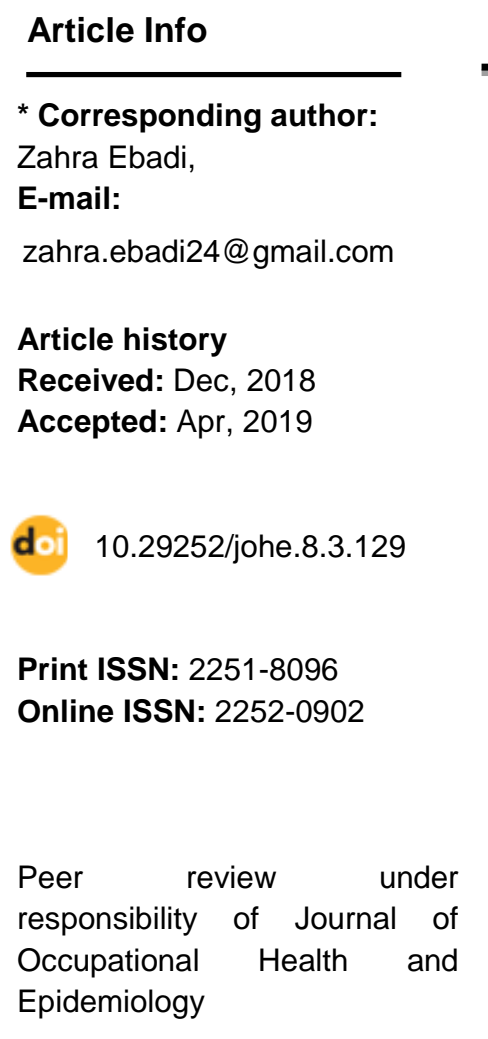

Citation: Ebadi Z. Association between prospective, retrospective memories and job performance of nurses in the city of Ahvaz, Iran, in 2016. JOHE 2019; 8(3):129-33.

\begin{abstract}
Background: Nurses continuously carry out several activities and need to develop certain cognitive mechanisms to be able to concentrate on their tasks. These activities and tasks affect and deal with the health and safety of people and their job performance. This study aims to investigate the association between prospective and retrospective memories and job performance of nurses in Imam Hospital, Ahvaz, Iran, in 2016.

Materials and Methods: This descriptive study was conducted on 150 nurses (67 males and 83 female) of Imam Hospital in Ahvaz based on an available sampling method. The Prospective and Retrospective Memory Questionnaire (PRMQ) determined the amount of prospective and retrospective memories, and job performance was evaluated using Steinmetz questionnaire. The collected data was analyzed using descriptive tests, analysis of variance, and Pearson correlation.

Results: The mean score of prospective memory, retrospective memory and job performance were $20.89( \pm 5.87), 19.43( \pm 5.12)$ and $52.46( \pm 8.14)$, respectively. Regression model showed that $22 \%$ of the variance in job performance is explained by prospective memory and retrospective memory.

Conclusion: We concluded prospective and retrospective memories were associated with the job performance of nurses working in Imam Hospital, Ahvaz, Iran. It is very important for the safety of the patients to improve the ability of the nurses who perform different tasks. In PM's tasks, they implemented strategies.
\end{abstract}

Keywords: Memory, Nursing, Job Performance

\section{Introduction}

Memory has been divided into retrospective memory and prospective memory. In what follows, retrospective memory and prospective memory will be considered in turn, focusing on the cognitive processes [1]. Prospective memory (ProM) allows us to make plans, retain them, and bring them back to one's consciousness at the right time and place. ProM is used for a variety of everyday tasks, including remembering to buy groceries, the route to home, picking up a child from daycare, keeping appointments, watching a bathtub so it does not overflow, taking medication at bedtime, and paying bills by the deadline. ProM is divided into several subdomains [2]. Although individual differences in processing speed, working memory, intelligence, and other cognitive functions were found to explain individual differences in retrospective memory (RetM), which less is known about their association with ProM [2].

The nursing environment is replete with eventbased and time-based prospective memory (PM) tasks (i.e. high prospective load) [3]. A nurse 's work environment places heavy demands on both time-based and event-based PM. Examples of such PM tasks include remembering to communicate with other staff, giving medications at specific times, checking for new orders, attending meetings, bringing patients' requests, continuous 
monitoring of changing information, and resuming tasks [3, 4]. Madadzadeh et al [5] reported that the workload is one of the most significant factors in nursing retention; measuring nursing workload reliability is the critical initial step to understanding the association between workload, nursing retention, and the safety and health of the patients [6].

In previous studies, different rates of nursing interruptions have been reported, from 6.3 interruptions per hour to 8 interruptions per shift [78]. Nursing environments in the real world make it possible to have higher probabilistic loads over longer periods of time, with more varied, more complex, and more continuous tasks, and more PM and emerging events that are delicate and revealing. In addition, research has shown that factors such as the importance of the PM task and rehearsal can affect PM performance [9-10]. The nurse's prospective burden increases interruptions in their job performances. Studies [11-13] have shown that interruptions in prospective memory are one of the key elements in adverse medical events that can affect nursing job performance. Despite the potential dangers of forgotten clinical work, few prospective memory researchers (PM) have the ability to deliver future tasks in the field of health care.

The aim of this study was to investigate and determine the association between prospective and retrospective memories and job performance of nurses in Imam Hospital, Ahvaz, Iran, in 2016.

\section{Materials and Methods}

This descriptive-correlational study was performed in 2016 on nurses working in health care centers of Imam Hospital, Ahvaz, Iran. Based on a previous study [14], the sample size was estimated to be 150 participants. The total number of nurses in both morning and evening shifts in different wards of the hospital were 350. According to Morgan Table, 165 people were selected, 15 of which were almost incomplete and were excluded from the research due to lack of reliability. Randomization was also based on the selection of nurses in different shifts. The participant nurses were selected using a random sampling method, taking sample size into consideration. All the nurse from the three hospitals were asked to complete the questionnaire. Inclusion criteria were: nurses employed in the hospitals of the Imam University of Medical Sciences, and at least two years of clinical experience. Finally, 150 subjects who met the inclusion criteria were enrolled in the study.

The specific inclusion criteria were: nurses who are working at primary and secondary health care levels, of all ages, both sexes, no previous history of visits to psychiatrists or psychologists, no medication or hospitalization due to mental illnesses in the previous year. The specific exclusion criteria included fatigue, lack of concentration, and unwillingness. First, the researcher attended the health care centers and obtained the ethical permission. Then, the study procedure was generally explained to the subjects. The participants consisted of male and female nurses, married and single with an age range of 25 to 40 years with different levels of education, from whom informed consents were obtained. The selection forms were completed through interviews, and if the nurses met the inclusion criteria, the prospective and retrospective memory questionnaire (PRMQ) and job performance questionnaires were handed to them.

The instrument used to measure the Retrospective-Prospective memory (PRMQ) was developed by Crawford et al [15]. It was used to examine the subjective measures of PM. The $P R M Q$ is a 16-item questionnaire. Each participant was asked to rate the frequency of the occurrence of each type of memory failure in their daily life on a 5-point scale. Confirmatory factor analysis indicated that the questionnaire included 16 items (Eight items for measuring prospective memory and eight items for measuring retrospective memory). The participants are asked to choose one of five possible responses under each question, with sign imprint. The response to this questionnaire is based on a 5-point Likert 5 scale ranging from always (score $=5$ ), most of the time $($ score $=4)$, sometimes (score $=3$ ), rarely (score $=$ 2 ), to never (score $=1)$. Questions 1, 3, 5, 7, 10, 12,14 , and16 are used for the measurement of prospective memory, and questions 2, 4, 6, 8, 9, 11,13 , and15 are used to measure retrospective memory. The minimum and maximum scores for each person are 16 and 80, respectively [15]. $P R M Q$ is a self-report questionnaire that, in addition to the retrospective and prospective memories, can be used to measure short-term and long-term memories and peripheral signs and symptoms of memory. Thus, although the main purpose of this self-report scale was the measurement of PM and $\mathrm{AM}$, each item can be classified into three dimensions. Crawford et al. [15] reported the validity of the overall scale and the retrospective and prospective memories to be $0.89,0.84$ and 0.80 , respectively, which indicates the high reliability of the questionnaire. Zare and Mostafaie [16] used a group of 382 participants and obtained the Cronbach $\alpha$ to be 0.38 . In this study Cronbach a was obtained to be 0.43 . 
The job performance questionnaire was developed by Patterson and translated by Seniority and Sugar CRM (quoted by Aslanpur Jokandan et al.) [17].

This is a one-dimensional survey which examines the performance of work using 15 questions. The responses to this questionnaire are based on a 5point Likert scale ranging from Always (Highest Score) to Never (Lowest Score). In order to measure the reliability of this questionnaire, Salehi used a method consisting alpha coefficients for $0 / 78$ and 0/86 at 0/001. Reliability of job performance in Zareie 's research was estimated to be $0 / 80$ and $0 / 87$ through Cronbach's alpha reliability [according to 18]. Reliability of job performance in this research was obtained to be0/71 through Cronbach's alpha.

Data were analyzed using SPSS 23. Descriptive statistics were used for demographic and clinical characteristics. Mean, standard deviation, and percentages were used for the classification and presentation of statistical data. A systematic regression analysis was used to determine the predictive power of prospective and retrospective memories and job performance of the participants. An analysis of variance and Pearson's correlation test were used to evaluate the association between variables, and the level of statistical significance was considered $\mathrm{P}<0.05$.

\section{Results}

The participants ranged in age from 25 to 40 years (M $30.70 \pm S D=6.13$ ). Clinical work experience levels varied from 2 to 15 years (Mean 7.5 years). Prior to conducting a regression analysis, an initial analysis was carried out to examine the normality of the variables' scores, the results of which are reported in Table 1.

Table 1: Normality of research variables

\begin{tabular}{cccc}
\hline Variables & Job performance & Retrospective memory & Prospective memory \\
\hline $\mathbf{Z}$ & 0.57 & 0.48 & 0.70 \\
\hline $\mathbf{P}$ & 0.28 & 0.26 & 0.20 \\
\hline
\end{tabular}

The mean and standard deviation were (20.89 $\pm 5.87)$ for predictive memory variables, (19.43 $\pm 5.12)$ for retrospective memory, and (52.46 \pm 8.14 ) for job performance.

The Pearson's correlation coefficient showed that there was a significant positive association between the pro-ret memories and job performance of nurses $(r=0.39, P=0.001 ; r=$ $0.37, P=0.01$, respectively).
As shown in table 4, according to stepwise regression results, prospective memory and retrospective memories were entered into the analysis. As shown in the table, $22 \%$ of the variance in job performance is explained by prospective memory and retrospective memory, and it means that pro-ret memories are only explaining $22 \%$ of the variance in predicting nurses' job performance.

Table 2: Results of regression analysis of predictive variables with step-by-step job performance of nurses Ahvaz City, Iran, in 2016

\begin{tabular}{|c|c|c|c|c|c|c|}
\hline \multirow{2}{*}{ Criteria variable } & \multirow{2}{*}{$\begin{array}{c}\text { Indexes } \\
\text { predict variable }\end{array}$} & \multirow{2}{*}{$\mathbf{R}$} & \multirow{2}{*}{$\mathbf{R 2}$} & \multirow{2}{*}{ FP } & \multicolumn{2}{|c|}{ Coefficient regression } \\
\hline & & & & & 1 & 2 \\
\hline \multirow{2}{*}{ Job performance } & Prospective memory & 0.39 & 0.15 & $\begin{array}{c}35.54 \\
p<0.001\end{array}$ & $\begin{array}{c}\beta=0.39 \\
\mathrm{t}=5.96 \\
\mathrm{P}=0.001\end{array}$ & \\
\hline & Retrospective memory & 0.47 & 0.22 & $\begin{array}{c}29.03 \\
p<0.001\end{array}$ & $\begin{array}{c}\beta=0.30 \\
\mathrm{t}=4.65 \\
\mathrm{P}=0.001\end{array}$ & $\begin{array}{c}\beta=0.28 \\
\mathrm{t}=4.38 \\
\mathrm{P}=0.001\end{array}$ \\
\hline
\end{tabular}

\section{Discussion}

This study was conducted with the aim of determining the association between prospective and retrospective memories and job performance of nurses. Overall, the findings showed that there was a significant positive association between the pro-ret memories and job performance of nurses. The results of the current research are consistent with those of Grundgeiger [9], which reported that nurses used deliberate reminders to manage their PM demands. PM in critical care might be supported by increasing the visibility of cues related to tasks; it helps nurses do their job in the best way.

Grundgeiger explained that the empirical manipulation of cues in a special-care unit simulation showed that visual cues increase the possibility that future tasks are performed [9]. As evidenced in the current research and supported by other studies, there is a significant correlation between nurses' PM and RM memories and their job performance [9, 13, 14]. 
A major challenge for private healthcare centers now is to seek ways to improve their employees' job performance and increase efficiency in their healthcare interventions [19]. Regarding this, nurses represent the workforce owing to the fact that they are the people who are directly related to the patients. Their job performance is essential in achieving organizational objectives in a consistent and effective way [20].

A study reported that workload and technological support are the most influential organizational variables on nurses' job performance [20]. Better preparing nurses to safely fulfil the task of medication administration in the clinical environment, with increased PM memory in the face of interruptions, could lead to a reduction in errors and concomitant improvements to patient safety [13]. Prospective memory plays an important role in successful task management by nurses $[9,10,21]$.

A nurse's work conditions place heavy demands on PM memory. Walders [21] studied interventions on prospective memory in the emergency agency, and memory events were a significant part of interventions. According to studies [21-22] conducted by various researches, the causes of nursing interruption are due to stress and organizational and personal factors that are significantly associated with the interaction of work. As mentioned earlier, nurses encounter various factors in their work environment, which reduce job performance, interrupts their PM memory, and causes a delay in their work process [22].

As noted above, the nurses have many kinds of work to do. Thus, it is very important to help them to improve their ability to handle PM tasks [9, 10, 21].

One limitation of this study was its cross-sectional nature. To be more realistic, the satisfaction of nurses and job performance indicators should be measured at frequent intervening times are needed to show the changes and the reliability of the findings. Another limitation was the presence of the researcher in the witnessing setting, which could affect the nurse's performance. Finally, tiredness and other problems could have affected job performance. It is necessary to conduct further research focusing on other hospitals and departments, age groups, personality differences, optimal ways to plan and implement activities, and strategies to improve the ability to remind nurses' activities to reduced interruption of PM-RM.

\section{Conclusion}

There is a significant positive association between pro-ret memories and job performance of nurses.
Considering the results of the present study and similar research and due to the importance of nurses' job performance, authorities should use ways to increase the facility of reminder so that both patients and nurses can enjoy the benefits. To this end, it is very important to improve the ability of nurses who perform different tasks for the safety of the patient. In PM's tasks, they implemented strategies.

\section{Acknowledgement}

This paper has been extracted from a research project approved by the Ethics Committee of the Payame Noor University of Khuzestan, Ahvaz, Iran (code: 5.14.895). We express our profound gratitude to the subjects, who participated in this study, as well as the authorities of Imam Hospital for their valuable support.

Conflict of interest: None declared.

\section{References}

1. Foley JA. Retrospective and prospective memory in healthy and cognitively impaired older adults: Using subjective and objective assessment. [PhD thesis]. Edinburgh, Scotland, United Kingdom: University of Edinburgh; 2007.

2. Uttl B, White CA, Cnudde K, Grant LM. Prospective memory, retrospective memory, and individual differences in cognitive abilities, personality, and psychopathology. PLoS One 2018; 13(3): e0193806.

3. Grundgeiger T, Sanderson PM, MacDougall HG, Venkatesh B. Distributed Prospective Memory: An Approach to Understanding How Nurses Remember Tasks. Proc Hum Factors Ergon Soc Annu Meet 2009; 53(11):759-63.

4. Fink N. Prospective Memory in the Nursing Enviroment: Effects of type of Prospective Task and Prospective Load. [MSc Thesis]. Clemson, South Carolina, United States: Clemson University; 2010.

5. Madadzadeh M, Barati H, Ahmadi Asour A. The association between workload and job stress among nurses in Vasei hospital, Sabzevar city, Iran, in 2016. Journal of Occupational Health and Epidemiology 2018; 7(2):83-9

6. Duffield C, Roche M, Merrick ET. Methods of measuring nursing workload in Australia. Collegian 2006; 13(1):16-22.

7. Biron $A D$, Lavoie-Tremblay $M$, Loiselle $C G$. Characteristics of work interruptions during medication administration. J Nurs Scholarsh 2009; 41(4):330-6.

8. Tucker AL, Spear SJ. Operational failures and interruptions in hospital nursing. Health Serv Res 2006; 41(3):643-62.

9. Grundgeiger $T$, Sanderson PM, Orihuela CB, Thompson A, MacDougall HG, Nunnink L, et al. Prospective memory in the ICU: the effect of 
visual cues on task execution in a representative simulation. Ergonomics 2013; 56(4):579-89.

10. Fink N, Pak R, Bass B, Johnston M, Battisto D. A Survey of Nurses Self-Reported Prospective Memory Tasks: What must they remember and what do they forget? Proceedings of the Human Factors and Ergonomics Society Annual Meeting 2010; 54(19):1600-4.

11. McCurdie T, Sanderson P, Aitken LM. Traditions of research into interruptions in healthcare: A conceptual review. Int J Nurs Stud 2017; 66:23-36.

12. Hayes C, Jackson D, Davidson PM, Power T. Medication errors in hospitals: a literature review of disruptions to nursing practice during medication administration. J Clin Nurs 2015; 24(21-22):3063-76.

13. Xiao $Y$, Rivera AJ, Probst A, Blocker $P$, Wolf $L$, Kellogg KM. Human Factors in the Wild: SOLUTIONS for Mitigating the Negative Impact of Interruptions in Healthcare (Discussion Panel). Proc Hum Factors Ergon Soc Annu Meet 2017; 61(1):630-4.

14. Caroselli C. Economic awareness of nurses: relationship to budgetary control. Nurs Econ 1996; 14(5):292-8.

15. Crawford JR, Smith G, Maylor EA, Della Sala S, Logie RH. The Prospective and Retrospective Memory Questionnaire (PRMQ): Normative data and latent structure in a large non-clinical sample. Memory 2003; 11(3):261-75.
16. Zare H, Alipour A, Mostafaie A. Standardization and Validity Retrospective MemoryProspective. Social Cognition 2014; 3(1):45-65.

17. Aslanpoor Jokandan M, Shahbazi F, Maleki R. The relationship between vigor, health and job performance in employees of the industrial company, Ahvaz. Journal of Social Psychology (New Findings in Psychology) 2011; 7(21):6537.

18. Dianatnasab M. Javidi $H$. Beqoli $H$. The Medition role of Job stress in the Relationship between psychological Capital and Personnels Performance of the Gachsaran Oil and Gaz Company Operatinh in Dashtgaz. Journal of Psychological Models and Methods 2014; 4(15):75-89.

19. Hee OC, Binti Kamaludin NH. Motivation and Job Performance among Nurses in the Private Hospitals in Malaysia. International Journal of Caring Sciences 2016; 9(1):342-7.

20. Top M. Organizational variables on nurses' job performance in Turkey: nursing assessments. Iran J Public Health 2013; 42(3):261-71.

21. Walders K. The Effect of interruptions on prospective memory in the emergency department. [MSc Thesis]. Columbus, Ohio, United States: Ohio State University; 2012.

22. Trybou J, Germonpre S, Janssens H, Casini A, Braeckman L, De Bacquer D, et al. Job-related stress and sickness absence among belgian nurses: a prospective study. J Nurs Scholarsh 2014; 46(4):292-301. 